\title{
RESOLVING ACYCLIC IMAGES OF NONORIENTABLE THREE-MANIFOLDS
}

\author{
DUSAN REPOVS' ${ }^{1}$ AND R. C. LACHER ${ }^{2}$
}

\begin{abstract}
We show that every I-LC $\mathbf{Z}_{2}$-homology 3-manifold (without boundary) which is an almost 1 -acyclic (over $\mathbf{Z}_{2}$ ) proper image of a nonorientable 3-manifold $M$ (without boundary) is a resolvable generalized 3-manifold. The analogous result for the case when $M$ is orientable was recently proved by $\mathrm{J}$. L. Bryant and $\mathrm{R}$. C. Lacher.
\end{abstract}

1. Introduction. A space $X$ is said to be locally simply connected (1-LC) if for every $x \in X$ and every neighborhood $U \subset X$ of $x$ there is a neighborhood $V \subset U$ of $x$ such that any loop in $V$ is null-homotopic in $U$. A compact subset $Y$ of an ANR $X$ is cell-like if for every neighborhood $U \subset X$ of $Y$ there is a neighborhood $V \subset U$ of $Y$ such that $V$ is contractible in $U$. A mapping $f$ of an ANR $M$ onto a space $N$ is cell-like (resp. monotone) if for every $x \in N, f^{-1}(x)$ is a cell-like set (resp. compact and connected). A mapping $f: X \rightarrow Y$ is proper if it is closed and if $f^{-1}(y)$ is compact for all $y \in Y$.

Let $R$ be a principal ideal domain. A metrizable space $X$ is an $R$-homology $n$-manifold (with respect to singular homology and without boundary) provided $H_{*}(X, X-\{x\} ; R) \cong H_{*}\left(\mathbf{R}^{n}, \mathbf{R}^{n}-\{0\} ; R\right)$ for each $x \in X$, where $H_{*}(; R)$ is the singular homology with coefficients in $R$. A generalized $n$-manifold is a euclidean neighborhood retract (ENR) that is also a Z-homology $n$-manifold. An $n$-dimensional resolution of a space $X$ is a pair $(M, f)$ where $M$ is an $n$-manifold without boundary and $f: M \rightarrow X$ is a proper, cell-like onto mapping.

J. L. Bryant and R. C. Lacher [2, Theorem 2] have proved that every locally contractible 1-acyclic over $\mathbf{Z}_{2}$ image $X$ of a 3-manifold $M$ without boundary admits a resolution. In particular, $X$ is a generalized 3-manifold. A refinement of their proof enabled them to omit the acyclicity hypothesis over a 0 -dimensional set, provided that $M$ was orientable [2, Theorem 3]. We prove that orientability is not necessary.

THEOREM 1.1. Let $f$ be a closed, monotone mapping from a 3-manifold $M$ without boundary onto a locally simply connected $\mathbf{Z}_{2}$-homology 3-manifold $X$. Suppose there is a 0 -dimensional set $Z \subset X$ such that $\check{H}^{1}\left(f^{-1}(x) ; \mathbf{Z}_{2}\right)=0$ for all $x \in X-Z$. Then the

Received by the editors February 14, 1983

1980 Mathematics Subject Classification. Primary 57P05, 57P99, 57N10; Secondary 57M35, 57M99, $54 \mathrm{~B} 15$

Key words and phrases. Generalized 3-manifolds, resolutions, neighborhoods of compacta in nonorientable 3-manifolds.

'Supported in part by a Research Council of Slovenia Graduate Fellowship and a Fulbright Travel Grant.

${ }^{2}$ Supported in part by NSF Grant No. MCS $81-02155$ 
set $C=\left\{x \in X \mid f^{-1}(x)\right.$ is not cell-like $\}$ is locally finite in $X$. Moreover, $X$ is a resolvable generalized 3-manifold.

As a corollary we obtain a partial converse in dimension 3 to the well-known fact that a cell-like upper semicontinuous decomposition $G$ of an $n$-manifold $M$ without boundary. always yields a generalized $n$-manifold (if $n \geqslant 4$ one must assume, in addition, that $M / G$ is finite dimensional) $[5,7]$.

COROLlaRY 1.2. Let $G$ be a 0 -dimensional upper semicontinuous decomposition of a closed 3-manifold $M$ such that $M / G$ is a 1-LC $\mathbf{Z}_{2}$-homology 3-manifold. Then the set $C=\{g \in G \mid g$ is not cell-like $\}$ is finite.

RemarK 1.3. Let $\pi: M \rightarrow M / G$ denote the quotient map, $H_{G}$ the collection of all nondegenerate elements of $G$, and $N_{G}$ their union.

(1) The Hopf maps or the Bing map [1] show that if $\pi\left(N_{G}\right)$ is a 1-manifold then all nondegenerate elements of $G$ may fail to be cell-like.

(2) Spine maps [1] show that $C=\{g \in G \mid g$ is not cell-like $\}$ may have any finite number of elements even when $C=H_{G}$.

(3) An easy modification of the construction of the Whitehead continuum [12] shows that all nondegenerate elements of $G$ may fail to be cellular even when $\pi\left(N_{G}\right)$ is a Cantor set and $G$ is cell-like. (For details see [11].)

2. Neighborhoods of compacta in nonorientable 3-manifolds. Under some additional hypotheses, Knoblauch's finiteness theorem [4, Theorem 1] extends to nonorientable 3-manifolds.

Proposition 2.1. For every closed nonorientable 3-manifold $M$ there exists an integer $K$ such that if $X_{1}, \ldots, X_{K+1} \subset M$ are pairwise disjoint compact sets and each $X_{i}$ has a neighborhood $U_{i} \subset M$ such that the inclusion-induced homomorphism $H_{1}\left(U_{i}-X ; \mathbf{Z}_{2}\right) \rightarrow H_{1}\left(M ; \mathbf{Z}_{2}\right)$ is trivial, then at least one $X_{i}$ has a neighborhood in $M$ which embeds in $\mathbf{R}^{3}$.

Proof. We shall suppress the $\mathbf{Z}_{2}$ coefficients from the notation. Let $X_{1}, \ldots, X_{n} \subset M$ be pairwise disjoint compact sets and suppose each $X_{i}$ has a neighborhood $U_{i} \subset M$ such that the inclusion-induced homomorphism $H_{1}\left(U_{i}-X_{i}\right) \rightarrow H_{1}(M)$ is trivial, and if $i \neq j$ then $U_{i} \cap U_{j}=\varnothing$. Let $X=\cup_{i=1}^{n} X_{i}$ and $U=\cup_{i=1}^{n} U_{i}$. Consider the following commutative diagram:

$$
\begin{aligned}
& \bigoplus_{i=1}^{n} H_{1}\left(U_{i}-X_{i}\right) \rightarrow \bigoplus_{i=1}^{n} H_{1}\left(U_{i}\right) \\
& \downarrow \cong \quad \downarrow \cong \\
& \begin{array}{ccccc}
H_{1}(U-X) \\
\downarrow
\end{array} \quad \rightarrow \quad \begin{array}{c}
H_{1}(U) \\
\downarrow
\end{array} \quad \rightarrow \quad \begin{array}{c}
H_{1}(U, U-X) \\
\end{array} \quad \rightarrow \cdots \\
& \cdots \rightarrow \quad H_{1}(M-X) \quad \rightarrow \quad H_{1}(M) \quad \rightarrow \quad H_{1}(M, M-X) \quad \rightarrow \cdots
\end{aligned}
$$

where the horizontal sequences are exact and $\Psi$ is the excision isomorphism. It is easy to see that the image of the inclusion-induced homomorphism $H_{1}(U) \rightarrow H_{1}(M)$ 
is the direct sum of the images of the inclusion-induced homomorphisms $H_{1}\left(U_{i}\right) \rightarrow$ $H_{1}(M), 1 \leqslant i \leqslant n$. So if we let $\beta_{1}=\operatorname{rank} H_{1}(M)$ then $n-\beta_{1}$ of the homomorphisms $H_{1}\left(U_{i}\right) \rightarrow H_{1}(M)$ are trivial. It follows by [8, Lemma (4.1)] that $n-\beta_{1}$ of the neighborhoods $U_{i}$ are orientable. Let $k(\tilde{M})$ be the Knoblauch number of the orientable 3-manifold double cover of $M$ [4, Theorem 1]. Since every orientable neighborhood lifts in $\tilde{M}$ to two (homeomorphic) copies, it follows that if $2\left(n-\beta_{1}\right)$ $>k(\tilde{M})$ then some $\tilde{X}_{i}$ has a neighborhood in $M$ which embeds in $\mathbf{R}^{3}$. We can now determine the number $K$ from the equation $2\left(K-\beta_{1}\right)=k(\tilde{M})$.

Proposition 2.2. Let $K$ be a compact connected subset of the interior of a 3-manifold $M$. Suppose $K$ does not separate its connected neighborhoods and, for every neighborhood $U \subset M$ of $K$ there exists a neighborhood $V \subset U$ of $K$ such that the inclusion-induced homomorphism $H_{1}\left(V-K ; \mathbf{Z}_{2}\right) \rightarrow H_{1}\left(U ; \mathbf{Z}_{2}\right)$ is trivial. Then $K=$ $\cap_{i=1}^{\infty} N_{i}$ where each $N_{i} \subset$ int $M$ is a compact 3-manifold with boundary satisfying the following properties:

(i) $N_{i+1} \subset$ int $N_{i}$;

(ii) $N_{i}$ is obtained from a compact 3-manifold $Q_{i}$ with a 2-sphere boundary by adding to $\partial Q_{i}$ a finite number of orientable ( solid) 1-handles;

(iii) the inclusion-induced homomorphism $H_{1}\left(\partial N_{i+1} ; \mathbf{Z}_{2}\right) \rightarrow H_{1}\left(N_{i} ; \mathbf{Z}_{2}\right)$ is trivial;

(iv) there is a homeomorphism $h_{i}: N_{i} \rightarrow N_{i}$ such that $h_{i} \mid \partial N_{i}=$ identity and $h_{i}\left(Q_{i}^{*}\right)=$ $Q_{i+1}$, where $Q_{i}^{*} \subset$ int $Q_{i}$ is formed by pushing $Q_{i}$ into int $Q_{i}$ along a collar of $\partial Q_{i}$.

REMARK 2.3. An examination of the proofs in [10] shows that the orientability hypothesis can be removed from all results in [10] if one uses Proposition 2.2 in place of $[9$, Theorem 2].

Proof of Proposition 2.2. By [13, Theorem 2], $K=\bigcap_{i=1}^{\infty} N_{i}$ where each $N_{i} \subset$ int $M$ is a compact 3-manifold with boundary satisfying (i) and (ii) above (the orientability of the 1-handles follows by [8, Lemma (4.1)]). By choosing an appropriate subsequence of $\left\{N_{i}\right\}$ we can satisfy (iii). We prove (iv). Let $K_{i} \subset$ int $Q_{i}$ be a spine of $Q_{i}$. Let $\hat{Q}_{i}$ be the closed 3-manifold we obtain by attaching a 3-cell to $\partial Q_{i}$. For each $i \geqslant 1, N_{1}=\left(N_{1} / K_{i}\right) \# \hat{Q}_{i}$ (the interior connected sum [3]). Since $N_{1}$ is nonorientable, it admits a unique normal, prime decomposition $N_{1}=M_{1} \# \cdots \# M_{n}$, $M_{i} \neq S^{2} \times S^{1}$ [3, Theorem (3.15) and Lemma (3.17)]. Consider normal, prime decompositions of $N_{1} / K_{1}$ and $\hat{Q}_{i}(i \geqslant 1)$. Since $N_{1} / K_{i}$ is clearly orientable, its normal, prime decomposition $N_{1} / K_{i}=A_{1} \# \cdots \# A_{p} \# B_{1} \# \cdots \# B_{q}$ may contain $p>0$ summands $A_{i}=S^{2} \times S^{1}$. On the other hand, $\hat{Q}_{1}$ is nonorientable (since $N_{1}$ is) so its normal prime decomposition $\hat{Q}_{i}=C_{1} \# \cdots \# C_{r}$ contains no $S^{2} \times S^{1}$ summands. By [3, Lemma (3.17)] we may replace each $A_{i}$ by $P=$ the nonorientable $S^{2}$-bundle over $S^{1}$ to get a normal, prime decomposition $N_{1}=P \# \cdots \# P \# B_{1} \#$ $\cdots \# B_{q} \# C_{1} \# \cdots \# C_{r}$ ( $p$ summands $P$ ) of $N_{1}$. It follows by the uniqueness of normal, prime decompositions that $p+q+r=n$ and that after a suitable permutation of the summands each $C_{i}$ is homeomorphic to some $M_{i}$. We may conclude that among any $n+1 \hat{Q}_{i}$ 's at least two have the same prime summands (up to a homeomorphism). By choosing an appropriate subsequence of $\left\{Q_{i}\right\}$ we may henceforth assume that for each $i \leqslant j$ there is a homeomorphism $s_{i j}: Q_{i} \rightarrow Q_{j}$. 
We first construct $h_{1}$. The identity on $\partial N_{1}$ induces a homeomorphism $t_{i j}^{\prime}$ : $\partial\left(N_{1} / K_{i}\right) \rightarrow \partial\left(N_{1} / K_{j}\right)$ for each $i \leqslant j$. Using Dehn's lemma we can extend $t_{i j}^{\prime}$ to a homeomorphism $t_{i j}: N_{1} / K_{i} \rightarrow N_{1} / K_{j}$. Finally, define $h_{i j}: N_{1} \rightarrow N_{1}$ by $h_{i j}(x)=s_{i j}(x)$ if $x \in Q_{i}$ and $h_{i j}(x)=t_{i j}(x)$ if $x \in N_{1}-Q_{i}$. Clearly, $h_{i j} \mid \partial N_{1}=$ identity and $h_{i j}\left(Q_{i}^{*}\right)=Q_{j}^{*}$. We define $h_{2}$ as the composition of $h_{12}$ and a homeomorphism of $N_{1}$ that is the identity outside a neighborhood of $\partial Q_{2}$ in $N_{2}$ and pushes $Q_{2}^{*}$ onto $Q_{2}$. We can get $h_{i}, i \geqslant 2$, in a similar way. For details see [11].

3. The proof of Theorem (1.1). We shall suppress the $\mathbf{Z}_{2}$ coefficients from the notation. Let $A=\left\{x \in X \mid \check{H}^{1}\left(f^{-1}(x)\right) \neq 0\right\}$. By [6, Theorem (4.1)], $A$ is locally finite in $X$. Let $B=\left\{x \in X \mid f^{-1}(x)\right.$ has no neighborhood embeddable in $\left.\mathbf{R}^{3}\right\}$. In order to show that $B$ is locally finite in $X$ it suffices, by Proposition 2.1 , to prove that for each $x \in X, f^{-1}(x)$ possess a neighborhood $U \subset M$ such that

$$
H_{1}\left(U-f^{-1}(x)\right) \rightarrow H_{1}(M)
$$

is trivial. So let $x \in X$. Since $A$ is locally finite there is a neighborhood $W \subset X$ of $x$ such that $W \cap A \subset\{x\}$. By hypothesis $X$ is $\mathrm{LC}^{l}$ so there is a connected neighborhood $W^{\prime} \subset W$ of $x$ such that any loop in $W^{\prime}$ is null-homotopic in $W$. Consider the following commutative diagram:

$$
\begin{array}{ccc}
H_{1}\left(f^{-1}\left(W^{\prime}\right)-f^{-1}(x)\right) & \stackrel{i_{*}^{\prime}}{\rightarrow} & H_{1}\left(f^{-1}(W)-f^{-1}(x)\right) \\
\left.\cong \downarrow f\right|_{*} & & \left.\simeq \downarrow f\right|_{*} \\
H_{1}\left(W^{\prime}-\{x\}\right) & \rightarrow & H_{1}(W-\{x\}) \\
\downarrow j_{*}^{\prime} & & \downarrow j_{*} \\
H_{1}\left(W^{\prime}\right) & \stackrel{i_{*}}{\rightarrow} & H_{1}(W)
\end{array}
$$

where the horizontal homomorphisms are induced by inclusions, $\left.f\right|_{*}$ is the Vietorismapping theorem isomorphism [7, 3.4], while $j_{*}$ and $j_{*}^{\prime}$ are the isomorphisms from the homology sequence of the pairs $(W, W-\{x\})$ and $\left(W^{\prime}, W^{\prime}-\{x\}\right)$, respectively. By hypothesis, $i_{*}=0$, hence $i_{*}^{\prime}=0$. Thus we may apply Proposition 2.1. By Proposition 2.2, $f^{-1}(x)$ is definable by (orientable) cubes with handles for all $x \in X-B$, so by [9, Theorem 3], $f^{-1}(x)$ has the 1-UV property. Since cubes with handles have no higher homotopy, each $f^{-1}(x)$ has the $\mathrm{UV}^{\infty}$ property and hence $C \subset B$ (cf. [7]). Therefore, $C$ is locally finite in $X$. In particular, $X-C$ is finite dimensional by [5]. A resolution of $X$ is now obtained by improving $f$ over the points of $C$. This is done similarly as in [2]. For details see [11].

\section{REFERENCES}

1. J. L. Bryant and R. C. Lacher, A Hopf-like invariant for mappings between odd-dimensional manifolds, Topology Appl. 8 (1978), 47-62.

2. $311-319$. , Resolving acyclic images of three-manifolds, Math. Proc. Cambridge Philos. Soc. 88 (1980),

3. J. Hempel, 3-manifolds, Ann. of Math. Studies, vol. 86, Princeton Univ. Press, Princeton, N.J., 1976.

4. T. Knoblauch, Imbedding compact 3-manifolds in $E^{3}$, Proc. Amer. Math. Soc. 48 (1975), 447-453. 
5. G. Kozlowski and J. J. Walsh, The cell-like mapping problem, Bull. Amer. Math. Soc. (N.S.) 2 (1980), 315-316.

6. R. C. Lacher, Some mapping theorems, Trans. Amer. Math. Soc. 195 (1974), 291-303.

7. Cell-like mappings and their generalizations, Bull. Amer. Math. Soc. 83 (1977), 495-552.

8. R. C. Lacher and D. R. McMillan, Jr., Partially acyclic mappings between manifolds, Amer. J. Math. 94 (1972), 246-266.

9. D. R. McMillan, Jr., Acyclicity in three-manifolds, Bull. Amer. Math. Soc. 76 (1970), 942-964.

10. D. R. McMillan, Jr. and T. L. Thickstun, Open three-manifolds and the Poincare conjecture, Topology 19 (1980), 313-320.

11. D. Repovš, Generalized three-manifolds with zero-dimensional singular set, $\mathrm{Ph}$. D. Thesis, Florida State University, Tallahassee, 1983.

12. J. H. C. Whitehead, A certain open manifold whose group is a unity, Quart. J. Math. Oxford Ser. 6 (1935), 268-279.

13. A. Wright, Mappings from 3-manifolds onto 3-manifolds, Trans. Amer. Math. Soc. 167 (1972), 479-495.

College of Mechanical Engineering, University of Ljubluana, 61001 Luubljana, P. O. Box 394, Yugoslavia

Department of Mathematics and Computer Science, florida State University, Tallahassee, FLORIDA 32306 